\title{
Control of methicillin-resistant Staphylococcus aureus in hospitals. An impossible dream?
}

The recent publication of the genome sequence of methicillin-resistant Staphylococcus aureus (MRSA) confirms the ability of $S$. aureus to acquire useful genes and to adapt to the physical environment and to the antibiotic milieu in which it can emerge and proliferate [1]. MRSA has been present in many UK hospitals and elsewhere for some years despite aggressive efforts to eradicate and control in many centres. The recent increase in prevalence in the face of these measures has resulted in controversy as to whether the effort involved in control represents a waste of resources and distracts microbiologists and others from more important issues [2-4]. Teare and Barrett argue that 'MRSA may not need any control. There is no convincing evidence, despite a vast body of literature, that it causes greater morbidity than methicillin sensitive $S$. aureus (MSSA), which is more prevalent' [2].

The most recent UK Guidelines published in 1998 attempted to establish a set of priorities for the use of resources in the control of MRSA especially where it is endemic [5]. Previous UK guidelines approached the issue assuming that MRSA was episodic and not endemic, and that, therefore, there was a good chance that it could be controlled and eradicated from the hospital or clinical unit where it was detected. Even in the short period since the publication of the latest MRSA recommendations in 1998, the situation has changed significantly in the UK, with the proportion of blood culture isolates causing bloodstream infection due to $S$. aureus now reaching $40 \%$ and in some areas even exceeding it. International comparisons indicate that there is considerable variation in the prevalence of MRSA in hospitals. Data from a recent study which analysed $3051 \mathrm{~S}$. aureus isolates from the European SENTRY antimicrobial surveillance programme revealed high prevalence rates in Portugal, Turkey, Italy, Spain and Portugal [6]. Even though this study collected isolates from specific centres and not nationally, the results confirmed that prevalence rates tend to be lower in Scandinavia, the Netherlands and Germany but higher in Southern Europe, as well as in the UK and Ireland. Does this indicate that control measures in those countries where MRSA prevalence is low are successful or that because MRSA has not become endemic the resources required to control and eradicate MRSA are considerably less and therefore more likely to be available? However, antimicrobial resistance is lower in the same countries, and not just amongst $S$. aureus, and this probably reflects difference in the use of antibiotics, differences in the approach to and compliance with infection control policies, and differences in the funding of health care.

The main arguments for specific measures to control MRSA in hospital lie in the mortality and morbidity associated with MRSA infection and the associated costs $[7,8]$. Any assessment of the clinical impact of MRSA infection is complicated by factors such as comorbidity (often greater in MRSA patients) and delays in the start of appropriate therapy (blind therapy of bacteraemia may not include a glycopeptide agent) when comparing outcome due to methicillin-sensitive and methicillin-resistant isolates. A recent prospective study of bacteraemia or bloodstream infection in Spain which took place over a 4-year period during an extensive outbreak confirmed that patients with MRSA are more likely to have significant co-morbidity as reflected in prolonged hospitalisation, but revealed that methicillin resistance was an independent risk factor for death [9]. Whilst the finding that the outcome from MRSA bacteraemia is worse that that from MSSA is at odds with other studies where mortality has been reported as similar, the evidence to date suggests that methicillin-resistant isolates are at least as virulent as methicillin-sensitive isolates.

Two recent reports argue that it is cost-effective to attempt to control the spread of MRSA. In one study conducted in Canada, patient-specific costs were used to determine the attributable cost of MRSA associated with excess hospitalisation and concurrent treatment, and following this, it was estimated that the cost associated with MRSA was $\$ 42-\$ 59$ million each year [8]. In a case-control study in a medical intensive care unit in a French University Hospital with a prevalence of $4 \%$ carriage amongst ICU patients, it was calculated that a $14 \%$ reduction in MRSA infection rates resulted in the control programme being economically beneficial [7].

Studies and reports of the successful control of MRSA are plagued by deficiencies in design, i.e., they are not 
controlled or are retrospective, and by differences in local epidemiology which make extrapolations to other hospitals or countries difficult. For example, successful efforts in the control of MRSA may be as much determined by the local epidemiology of MRSA and in particular whether MRSA is episodic/epidemic or endemic than by the level of resources allocated or the stringency of the measures initiated. Inadequate isolation facilities, the need to maximise bed usage and modern methods of patient management have been cited as reasons why a comprehensive MRSA programme is impossible [4]. Nonetheless, most would agree that specific efforts are certainly indicated in critically ill patients at risk of invasive infection including bloodstream infection. In one study to reduce the risk of ventilator-associated pneumonia, the routine application of mupirocin together with universal precautions resulted in a significant decrease in pneumonia [10]. However, this was a retrospective analysis and the emergence of mupirocin resistance, particularly high-level resistance, amongst strains of MRSA might be accelerated by such a policy were it to become routine. Enhanced surveillance and enhanced infection control measures together with molecular typing of MRSA isolates to help indicate patterns of spread resulted in a decrease in the annual frequency of hospital-acquired MRSA in one US hospital [11].

Those who believe that measures to control MRSA are neither cost-effective nor clinically appropriate because they deflect attention from other infection control issues may not in reality be very different in their actual approach to those who believe in a specific MRSA control programme. Control of MRSA must not be seen as separate from measures to prevent nosocomial infection generally but rather incorporated as part of a hospital-wide strategy directed in those clinical areas where any improvements are likely to be greatest in terms of their clinical impact. Most would agree that enhanced hand hygiene has a significant role to play in reducing nosocomial infection. Pittet et al. monitored overall compliance with hand hygiene during 20000 opportunities as part of routine patient care in a teaching hospital in Geneva [12]. Compliance improved progressively after a series of measures and was accompanied by a decrease in the prevalence of nosocomial infection from $16.9 \%$ to 9.9\% as well as a decrease in MRSA transmission rates from 2.16 to 0.93 episodes per 10000 patient-days. This study demonstrated what can be done when significant resources, both human and educational, are targeted at hospital-wide interventions.

There is a consensus that the careful and appropriate use of antibiotics is critical in reducing the pressure on the emergence of antibiotic resistance. Whilst the relationship between antimicrobial use and the emergence and spread of MRSA is undoubtedly complex the impact that more appropriate use may have in relieving antimicrobial pressure has probably been under-esti- mated. In a study involving 50 hospitals in Belgium, the number of new nosocomial MRSA-colonised or -infected patients was correlated with the use of various antimicrobial classes, and multivariate analysis showed that the incidence of MRSA increased with increasing use of ceftazidime and cefsulodin [13]. In Denmark there was a rise in the frequency of MRSA isolation in the late 1960s and early 1970s, but up to the early 1990 s this had remained at levels $<1 \%$ [14]. It is difficult to argue against the view of the authors that this is largely attributable to tight control of antibiotic use there, especially the use of cephalosporins, as is the case throughout the Scandinavian countries. A recent prevalence survey of MRSA conducted throughout Ireland, where there are two separate health care systems, and which included a survey of control measures, revealed that less than half of acute hospitals in the Republic of Ireland had a written antibiotic policy compared with $95 \%$ in the North of Ireland where the prevalence of MRSA was less [15]. However, the presence of a policy needs to be accompanied by an on-going education programme and by audit to ensure compliance. In addition, the physical environment of patients, both in terms of adequate space and hygiene, needs to be considered. A recent intervention study which included increased domestic cleaning time, with emphasis on removal of dust by vacuum cleaning, and allocation of responsibility for the routine cleaning of shared medical equipment - resulted in fewer colonised patients and less environmental contamination [16].

Whilst the total eradication of MRSA in many hospitals where it is now endemic may not be feasible, control measures - including surveillance and eradication - is, and should be, an objective - particularly in high-risk areas. Such areas include the intensive care unit, neonatal intensive care unit, clinical areas where there are compromised patients such as the ICU, and orthopaedic/trauma wards where many patients have breaks in the skin and multiple devices in situ. MRSA control should be a component of institution-based infection control programmes and not a stand-alone entity and seen as something that consumes scarce resources. Improved surveillance of nosocomial infection, enhanced hygiene, better use of antimicrobial agents and more effective environmental cleaning regimens should be the goal of all those involved in the control of hospital-acquired infection irrespective of their individual perspective on MRSA control.

HILARY HUMPHREYS

Department of Clinical Microbiology, Royal College of Surgeons in Ireland and Beaumont Hospital,

Dublin, Ireland

(e-mail: hhumphreys@rcsi.ie)

\section{References}

1. Kuroda M, Ohta T, Uchiyama I et al. Whole genome sequencing of methicillin-resistant Staphylococcus aureus. Lancet 2001; 357: 1225-1240. 
2. Teare EL, Barrett SP. Stop the ritual of tracing colonised people. BMJ 1997; 314: 665-666.

3. Farrington M, Redpath C, Trundle C, Coomber S, Brown NM. Winning the battle but losing the war: methicillin-resistant Staphylococcus aureus (MRSA) infection at a teaching hospital. $Q J$ Med 1998; 91: 539-548.

4. Barrett SP, Mummery RV, Chattopadhyay B. Trying to control MRSA causes more problems than it solves. J Hosp Infect 1998; 39: 85-93.

5. Report of combined Working Party of the British Society for Antimicrobial Chemotherapy, The Hospital Infection Society and the Infection Control Nurses Association. Revised guidelines for the control of methicillin-resistant Staphylococcus aureus infection in hospitals. J Hosp Infect 1998; 39: 253290.

6. Fluit AC, Wielders CLC, Verhoef J, Schmitz F-J. Epidemiology and susceptibility of 3,051 Staphylococcus aureus isolates from 25 University hospitals participating in the European SENTRY Study. J Clin Microbiol 2001; 39: 3727-3732.

7. Chaix C, Durand-Zaleski I, Alberti C, Brun-Buisson C. Control of endemic methicillin-resistant Staphylococcus aureus. A costbenefit analysis in an intensive care unit. JAMA 1999; 282: $1745-1751$

8. Kim T, Oh PI, Simor AE. The economic impact of methicillinresistant Staphylococcus aureus in Canadian hospitals. Infect Control Hosp Epidemiol 2001; 22: 99-104.

9. Romero-Vivas J, Rubio M, Fernandez C, Picazo JJ. Mortality associated with nosocomial bacteremia due to methicillinresistant Staphylococcus aureus. Clin Infect Dis 1995; 21: 1417-1423.

10. Rumbak MJ, Cancio MR. Significant reduction in methicillinresistant Staphylococcus aureus ventilator-associated pneumonia associated with the institution of a prevention protocol. Crit Care Med 1995; 23: 1200-1203.

11. Hartstein AI, Denny MA, Morthland VH, LeMonte AM, Pfaller MA. Control of methicillin-resistant Staphylococcus aureus in a hospital and an intensive care unit. Infect Control Hosp Epidemiol 1995; 16: 405-411.

12. Pittet D, Hugonnet S, Harbarth $\mathrm{S}$ et al. Effectiveness of a hospital-wide programme to improve compliance with hand hygiene. Lancet 2000; 356: 1307-1312.

13. Crowcroft NS, Ronveaux O, Monnet DL, Mertens R Methicillin-resistant Staphylococcus aureus and antimicrobial use in Belgian hospitals. Infect Control Hosp Epidemiol 1999; 20: $31-36$.

14. Rosdahl VT, Knudsen AM. The decline of methicillin resistance among Danish Staphylococcus aureus strains. Infect Control Hosp Epidemiol 1991; 12 83-88.

15. North/South Study of MRSA in Ireland, 1999. Dublin, Ireland, Department of Health and Children. 2000. (www.doh.ie./ publications/mrsa99.html)

16. Rampling A, Wiseman S, Davis L et al. Evidence that hospital hygiene is important in the control of methicillin-resistant Staphylococcus aureus. J Hosp Infect 2001; 49: 109-116. 